\title{
The way to improve the accuracy of control of maneuvering of the vessel by as- sessing the abscissa of the center of gravity
}

\author{
I. L. Surinov \\ National university "Odessa maritime academy”, Odessa, Ukraine \\ Corresponding author. E-mail: the_best_igor@ukr.net
}

Paper received 10.09.20; Accepted for publication 24.09.20.

\begin{abstract}
https://doi.org/10.31174/SEND-NT2020-238VIII29-14
\end{abstract}
\begin{abstract}
This article presents an algorithm that allows to quickly determine the center of gravity of a vessel. When considering maneuvering control issues, the vessel is considered as a point, the mass of which is concentrated at the center of gravity. The origin of coordinates is placed at the point of intersection of the diametric plane and the mid-frame. The error in determining the location is commensurate with the value of the abscissa of the center of gravity, and sometimes much less. Accurate determination of the coordinates of the center of gravity will reduce the error when finding the ship's position.
\end{abstract}

Keywords: abscissa of centre of gravity, static moment of displacement of the vessel; ship landing assessment; displacement; maneuverability.

Introduction. Any sea vessel is a complex structure, the design of which requires the solution of numerous and very diverse issues arising on the way of developing a project from the most general concept to creating a set of drawings transferred to a shipbuilding enterprise. Practice has shown that this entire path cannot be passed at once, in one step, therefore, in order to make full-fledged and well-grounded decisions, it is required to overcome it gradually, step by step or step by step, checking and analyzing the results obtained at each stage, and then refining and correcting them when going to the next stage.

The issue related to the determination of the masses and coordinates of the center of gravity $(\mathrm{CG})$ of the vessel is one of the most important issues in the design of the vessel, since many important qualities of the vessel depend on it: seaworthiness. During the design process, it is necessary to determine the mass and coordinates of the ship's CG. The greatest difficulty for the designer is the determination of the mass and coordinates of the $\mathrm{CG}$ of empty ship [1].

Brief overview of related publications. In publication [2] was developed an algorithm and a block diagram of the navigation device for calculation the corrections of the antenna coordinates for recalculation to the center of gravity of the vessel. For a more accurate operation of the block diagram, an algorithm for calculating the $\mathrm{CG}$ is presented in this paper.

In paper [3], the authors developed an algorithm for determining the pivot point using a Doppler lag. In order to accurately determine the distance of the pivot point from the CG, you also need to know the exact coordinates of the center of gravity.

Purpose: 1) increasing the accuracy of determining the position of the vessel; 2) refinement of the calculation method for determining the abscissa of the pivot point.

When considering management issues, maneuvering is considered as a point. The mass of which is concentrated at the center of gravity. The origin of coordinates is placed at the point of intersection of the diametrical plane and the mid-frame.

The error in determining the location is commensurate with the value of the abscissa of the $\mathrm{CG}$, and sometimes much less.

Materials and methods. At the initial stages of design, empirical dependencies can be used to determine the lightweight of a ship, which are obtained by statistical processing of data on the mass load of built and designed ships [1].

The CG coordinate of any object relative to any selected base plane is the ratio of the sums of the statistical moments of the individual elements into which the object under consideration is divided to the sum of the weights of all elements, that is, to the weight of the entire object [4]. To determine the abscissa of the CG $\left(x_{g}\right)$ of the vessel, it is necessary to divide the static moment of the vessel's displacement relative to the midsection $\left(M_{x}\right)$ by the total displacement $(\Delta)$ :

$$
x_{g}=\frac{M_{x}}{\Delta} \text {. }
$$

The static moment of the ship's displacement relative to the midsection $\left(M_{x}\right)$ consists of the sum of the moments created by the ship's light $\left(M_{x_{0}}\right)$, cargo $\left(M_{x_{i}}\right)$ and reserves $\left(M_{x_{j}}\right)$, as given in formula (2).

$$
M_{x}=M_{x_{0}}+M_{x_{i}}+M_{x_{j}} \text {. }
$$

To determine the applicability of the CG $\left(z_{g}\right)$ of a loaded vessel, it is necessary to divide the static moment of loads relative to the main plane $\left(M_{z}\right)$ by the total displacement $(\Delta)$. However, it is necessary to take into account the presence of a free surface of the liquid in tanks and cisterns. For this, correction moments $\left(\Delta m_{h}\right)$ are introduced. The corrected CG applicate is given in formula 3 .

$$
z_{g}=\frac{M_{z}+\sum_{j=1}^{m} \Delta m_{h}}{\Delta}
$$

The static moment of loads relative to the main plane ( $M_{z}$ ) is found as the sum of the moments from the masses of the light ship $\left(M_{z_{0}}\right)$, cargo $\left(M_{z_{i}}\right)$ and stores $\left(M_{z_{j}}\right.$ ):

$$
M_{z}=M_{z_{0}}+M_{z i}+M_{z j}
$$


The presence of a free surface of the liquid in ship's tanks leads to a decrease in the transverse metacentric height and, therefore, deteriorates the lateral stability of the ship. The larger the dimensions of the tank, especially the width, the greater the effect of the free surface of the liquid. If there is a free surface of liquid in several tanks, then their total effect can be so great that the vessel will lose stability. It is not necessary that the vessel capsizes, but it can get permanent heel. The amount of roll will depend on the specific parameters of the lateral stability of the vessel. The influence of the free surface of the liquid is calculated as the correction by which the initial transverse metacentric height decreases and is called the correction for the influence of the free surface of the liquid $\left(\Delta m_{h}\right)[5]$.

If a ship's tank is $100 \%$ filled with any liquid, then it is considered that there is no free surface in this tank and its effect on the stability of the ship can be considered in the same way as the effect of any other cargo having a fixed center of gravity.

Let us consider the physical meaning of the influence of a free surface. If, under the influence of external influence, the liquid starts to move, then a moment of inertia arises at the free surface of the liquid. The moment of inertia of the free surface of the liquid, relative to its own axis, leads to the appearance of a virtual center of gravity at a certain height above its surface. In this case, the effect of the free surface on the lateral stability of the vessel will be the same as if the cargo with the same weight was moved from the tank to the point of the virtual center of gravity. Figure 1 shows the movement of fluid due to the presence of free space in the tank and the emergence of a virtual center of gravity [5].



Fig 1. Influence of the free surface of the liquid on the stability of the vessel

Figure 1 shows that if the ship tilts at a certain angle, then part of the liquid will move, and as a result, the shape of the volume of the liquid in the tank will change. The center of gravity of the liquid will move to point $\mathrm{g} 1$. Since the center of gravity of the liquid is at the same time its center of magnitude, the displacement gg1 can be considered the curve of the center of magnitude. Obviously, if we consider this movement by analogy with the inclination of a vessel, then a change in the center of the magnitude of its position will cause the appearance of a transverse metacentre at point $\mathrm{m}$ and gm is the transverse metacentric radius, which can be calculated by the formula:

$$
g m=\frac{i}{v},
$$

where: $i$ - moment of liquid; inertia; $v$ - liquid volume.

On the vessel, to facilitate the calculation of the free surface correction, the so-called free surface moment is indicated in the Ship Stability Information for each ship's tank. Let's designate it with the letter I. As a rule, it is indicated for the density of the liquid in the tank equal to
$1,000 \mathrm{t} / \mathrm{m}^{3}$. If the actual density of the liquid differs from the calculated one, then the value of I must be multiplied by the actual density [5].

The correction for the free surface of the liquid is calculated by the formula:

$$
\Delta m_{h}=I \times \frac{q}{D},
$$

where: I - moment of free surface of a liquid in a ship's tank; q - density of the liquid in the tank; D - weight displacement of a vessel.

The ordinate of the center of gravity, as a rule, is not calculated, since the loads are stacked symmetrically with respect to the center plane and for them $y_{i}=0$. In any case, the total moment $M_{y}$ from asymmetrically accepted loads should be small enough so that there is no noticeable heel angle, which is unacceptable during normal operation [8].

To commence the calculations, it is necessary to introduce the concept of the most static moment of the area of any figure. The static moment of the area is the sum of the products of elementary areas dA by the distance from them to this axis, spread over the entire area [6]. Consider a cross-section (flat figure) of arbitrary shape with area A (Fig. 2). Let's select the elementary area dA in it and set the coordinate system. The coordinates of the site will be designated as $\mathrm{X}$ and $\mathrm{Y}$, respectively. The static moment of an elementary site relative to the $\mathrm{X}$ axis is given in formula 7, and relative to the $\mathrm{Y}$ axis - in formula 8 [7].

$$
\begin{aligned}
& d S_{x}=y d A, \\
& d S_{y}=x d A .
\end{aligned}
$$

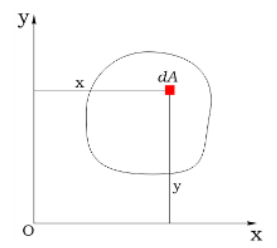

Fig 2. Determination of the static moment of a flat figure

Summing up the expressions over the entire area of the figure, we get, respectively:

$$
\begin{aligned}
& S_{x}=\int_{A} y d A, \\
& S_{y}=\int_{A} x d A .
\end{aligned}
$$

To find the center of gravity of a complex-shaped body, it is necessary to mentally break the body into parts of a simple shape and determine the location of the centers of gravity for them. For bodies of simple shape, the center of gravity is determined using their symmetry. So, the center of gravity of a homogeneous disk and a ball is located in their center, a homogeneous cylinder at a point in the middle of its axis; a homogeneous parallelepiped at the intersection of its diagonals, etc. For all homogeneous bodies, the center of gravity coincides with the center of symmetry. The center of gravity can be outside the body, for example, at the ring.

Having determined where the centers of gravity of individual parts of the body are located (Fig. 3), they proceed to find the location of the center of gravity of the body as a whole. The body is represented as a system of 
material points. Moreover, each point has the mass of its part of the body and is located in its center of gravity [7].

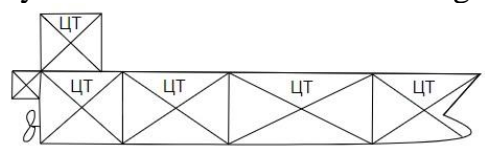

Fig 3. Determination of the CG of individual parts of the ship's hull

In three-dimensional space, the coordinates of the center of gravity for a rigid body are found as given in formula 11 .

$$
\left\{\begin{array}{c}
x_{c}=\frac{\sum_{i} \Delta m_{i} x_{i}}{m} \\
y_{c}=\frac{\sum_{i} \Delta m_{i} y_{i}}{m} \\
z_{c}=\frac{\sum_{i} \Delta m_{i} z_{i}}{m}
\end{array}\right\}
$$

where: $m$ - boy mass; $x_{i}$ - coordinates on the $\mathrm{X}$-axis of the elementary mass $\Delta m_{i} ; y_{i}$ - coordinates on the $\mathrm{Y}$ axis of the elementary mass $\Delta m_{i} ; z_{i}$ - coordinates on the Z-axis of the elementary mass $\Delta m_{i}$.

Results. Understanding the general principles of determining the static moment of inertia and the coordinates of the center of gravity of any body, you can start calculating the coordinates of the center of gravity of the vessel. This is best done in tabular form. To simplify the work, calculations are made either in Excel (this program is developed and provided in this article), or in a special program that is available on the ship.
The first stage - we determine the moments $\left(M_{x_{0}}\right.$ and $M_{z_{0}}$ ) and displacement ( $\left.\Delta_{0}\right)$ created by the light ship.

Light displacement - the displacement of a vessel in tons without cargo, fuel, lubricating oil, ballast, fresh, boiler water in tanks, waste water, ship's stores, as well as without passengers, crew and their belongings, but with water, fuel, oil in boilers, engines and pipelines [9]. These data are indicated in the document "Information on the stability of the vessel", as well as the coordinates of the CG of the vessel when empty.

To determine the static moments of a light vessel, it is necessary to multiply the light vessel displacement $\left(\Delta_{0}\right)$ by the coordinates of the center of gravity:

$$
\left\{\begin{array}{l}
M_{x_{0}}=\Delta_{0} \times x_{0} \\
M_{z_{0}}=\Delta_{0} \times z_{0}
\end{array}\right\}
$$

The second stage - we calculate the moments and displacement created by the reserves. Reserves include all fuels, oil and fresh water. The value of the abscissa of the coordinates of the tank or tanker CG is considered positive if it is located from the midsection and negative - if it is in the stern. The general formula for calculating the moment is:

$$
\left\{\begin{array}{l}
M_{x_{j}}=\sum_{j=1}^{m} P_{j} \times x_{j} \\
M_{z j}=\sum_{j=1}^{m} P_{j} \times z_{j}
\end{array}\right\}
$$

The calculation of ship's stores is shown in Table 1. In the same table, we determine the correction moment of the free surface, which will be taken into account when

\begin{tabular}{|c|c|c|c|c|c|c|c|}
\hline \multirow{2}{*}{$\begin{array}{l}\text { Stores } \\
\text { type }\end{array}$} & \multirow{2}{*}{ Stores location } & \multirow{2}{*}{$\frac{\overrightarrow{0}}{000}+$} & \multicolumn{2}{|c|}{$\begin{array}{c}\text { Coordinates CG, } \\
\mathrm{m}\end{array}$} & \multicolumn{2}{|c|}{ Moments, tm } & \multirow{2}{*}{ 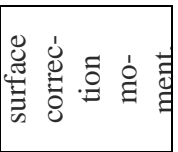 } \\
\hline & & & $x_{j}$ & $z_{j}$ & $M_{x_{j}}$ & $M_{z_{j}}$ & \\
\hline \multirow{5}{*}{$\begin{array}{l}\overline{0} \\
\sum^{2} \\
\overline{0} \\
0 \\
\dot{0}\end{array}$} & Tank №1 & $P_{11}$ & $x_{11}$ & $z_{11}$ & $P_{11} \times x_{11}$ & $P_{11} \times z_{11}$ & $\Delta m_{h_{11}}$ \\
\hline & Tank №2 & $P_{12}$ & $x_{12}$ & $z_{12}$ & $P_{12} \times x_{12}$ & $P_{12} \times z_{12}$ & $\Delta m_{h_{12}}$ \\
\hline & Tank №3 & $P_{13}$ & $x_{13}$ & $z_{13}$ & $P_{13} \times x_{13}$ & $P_{13} \times z_{13}$ & $\Delta m_{h_{13}}$ \\
\hline & $\ldots$ & $\ldots$ & $\ldots$ & $\ldots$ & $\ldots$ & $\ldots$ & $\ldots$ \\
\hline & Tank № n & $P_{1 n}$ & $x_{1 n}$ & $z_{1 n}$ & $P_{1 n} \times x_{1 n}$ & $P_{1 n} \times z_{1 n}$ & $\Delta m_{h_{1} n}$ \\
\hline \multirow{5}{*}{ 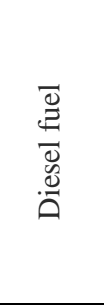 } & Tank №1 & $P_{21}$ & $x_{21}$ & $z_{21}$ & $P_{21} \times x_{21}$ & $P_{21} \times z_{21}$ & $\Delta m_{h_{21}}$ \\
\hline & Tank № 2 & $P_{22}$ & $x_{22}$ & $z_{22}$ & $P_{22} \times x_{22}$ & $P_{22} \times z_{22}$ & $\Delta m_{h_{22}}$ \\
\hline & Tank № 3 & $P_{23}$ & $x_{23}$ & $z_{23}$ & $P_{23} \times x_{23}$ & $P_{23} \times z_{23}$ & $\Delta m_{h_{23}}$ \\
\hline & $\ldots$ & $\ldots$ & $\ldots$ & $\ldots$ &  & & $\ldots$ \\
\hline & Tank № n & $P_{2 n}$ & $x_{2 n}$ & $z_{2 n}$ & $P_{2 n} \times x_{2 n}$ & $P_{2 n} \times z_{2 n}$ & $\Delta m_{h_{2 n}}$ \\
\hline$\ldots$ & $\ldots$ & $\ldots$ & $\ldots$ & $\ldots$ & $\ldots$ & $\ldots$ & $\ldots$ \\
\hline \multirow{2}{*}{ 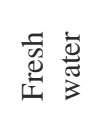 } & Tank №1 & $P_{m 1}$ & $x_{m 1}$ & $z_{m 1}$ & $P_{m 1} \times x_{m 1}$ & $P_{m 1} \times z_{m 1}$ & $\Delta m_{h_{m 1}}$ \\
\hline & Tank №2 & $P_{m 2}$ & $x_{m 2}$ & $z_{m 2}$ & $P_{m 2} \times x_{m 2}$ & $P_{m 2} \times z_{m 2}$ & $\Delta m_{h_{m 2}}$ \\
\hline
\end{tabular}
determining the $\mathrm{CG}$ applicates.

Table 1. Calculation of static movements created by ship's stores 


\begin{tabular}{|c|c|c|c|c|c|c|c|}
\hline & Tank №3 & $P_{m 3}$ & $x_{m 3}$ & $z_{m 3}$ & $P_{m 3} \times x_{m 3}$ & $P_{m 3} \times z_{m 3}$ & $\Delta m_{h_{m 3}}$ \\
\cline { 2 - 7 } & $\ldots$ & $\ldots$ & $\ldots$ & $\ldots$ & $\ldots$ & $\ldots$ & $\ldots$ \\
\cline { 2 - 7 } & Tank №n & $P_{m n}$ & $x_{m n}$ & $z_{m n}$ & $P_{m n} \times x_{m n}$ & $P_{m n} \times z_{m n}$ & $\Delta m_{h_{m n}}$ \\
\hline \multirow{2}{*}{ Total } & $\sum_{j=1}^{m} P_{j}$ & & & $\sum_{j=1}^{m} M_{x_{j}}$ & $\sum_{j=1}^{m} M_{z_{j}}$ & $\sum_{j=1}^{m} \Delta m_{h_{j}}$ \\
\hline
\end{tabular}

The third stage - we find the moments and displacement, which are created by the transported cargo. Weights that are located forward of the midsection have a positive abscissa sign, and weights that are located aft have a negative sign. The general formula for finding the moments is:

$$
\left\{\begin{array}{l}
M_{x_{i}}=\sum_{i=1}^{n} Q_{i} \times x_{i} \\
M_{z_{i}}=\sum_{i=1}^{n} Q_{i} \times z_{i}
\end{array}\right\}
$$

The calculation of the displacement and the moments created by the weights are shown in Table 2 .

Table 2. Calculation of displacement and moments generated by weights

\begin{tabular}{|c|c|c|c|c|c|}
\hline \multirow{2}{*}{ Cargo space } & \multirow{2}{*}{$\begin{array}{c}\text { Weight of } \\
\text { cargo, } \mathrm{n}\end{array}$} & $x_{i}$ & $z_{i}$ & $M_{x_{i}}$ & $M_{z_{i}}$ \\
\cline { 3 - 6 } & $Q_{11}$ & $x_{11}$ & $z_{11}$ & $Q_{11} \times x_{11}$ & $Q_{11} \times z_{11}$ \\
\hline Hold № 1 & $Q_{12}$ & $x_{12}$ & $z_{12}$ & $Q_{12} \times x_{12}$ & $Q_{12} \times z_{12}$ \\
\hline Hold № 2 & $Q_{13}$ & $x_{13}$ & $z_{13}$ & $Q_{13} \times x_{13}$ & $Q_{13} \times z_{13}$ \\
\hline Hold №3 & $\ldots$ & $\ldots$ & $\ldots$ & $\ldots$ & $\ldots$ \\
\hline .. & $Q_{1 m}$ & $x_{1 m}$ & $z_{1 m}$ & $Q_{1 m} \times x_{1 m}$ & $Q_{1 m} \times z_{1 m}$ \\
\hline Hold № m & $Q_{21}$ & $x_{21}$ & $z_{21}$ & $Q_{21} \times x_{21}$ & $Q_{21} \times z_{21}$ \\
\hline Twindeck № 1 & $Q_{22}$ & $x_{22}$ & $z_{22}$ & $Q_{22} \times x_{22}$ & $Q_{22} \times z_{22}$ \\
\hline Twindeck №2 & $Q_{23}$ & $x_{23}$ & $z_{23}$ & $Q_{23} \times x_{23}$ & $Q_{23} \times z_{23}$ \\
\hline Twindeck №3 & $\ldots$ & $\ldots$ & $\ldots$ & $\ldots$ & $\ldots$ \\
\hline$\ldots$ & $Q_{2 m}$ & $x_{2 m}$ & $z_{2 m}$ & $Q_{2 m} \times x_{2 m}$ & $Q_{2 m} \times z_{2 m}$ \\
\hline Twindeck № m & $\sum_{i=1}^{n} Q_{i}$ & & & $\sum_{i=1}^{n} M x_{i}$ & $\sum_{i=1}^{n} M_{z_{i}}$ \\
\hline Total & & & & $\ldots$ & \\
\hline
\end{tabular}

The fourth stage - we determine the total displacement $(\Delta)$. It consists of the ship's light displacement $\left.\Delta_{0}\right)$, the mass of the ship's stores $\left(P_{j}\right)$ and the weight of the total cargo $\left(Q_{i}\right)$. These calculations are shown in Formula 15.

$$
\Delta=\Delta_{0}+P_{j}+Q_{i}
$$

The fifth stage - using formula 1, we determine the abscissa of the ship's center of gravity and using formula
3 - the applicator's center of gravity.

Conclusions. All the work done can be represented in the form of a block diagram in Fig. 4. The abscissa CG is used to assess the ship's landing, and the applicate is used to assess the stability of the ship.

Knowing the coordinates of the CG will help in the future to recalculate the parameters of the satellite dish to the center of gravity of the vessel, which will increase the accuracy of determining the position of the vessel. 


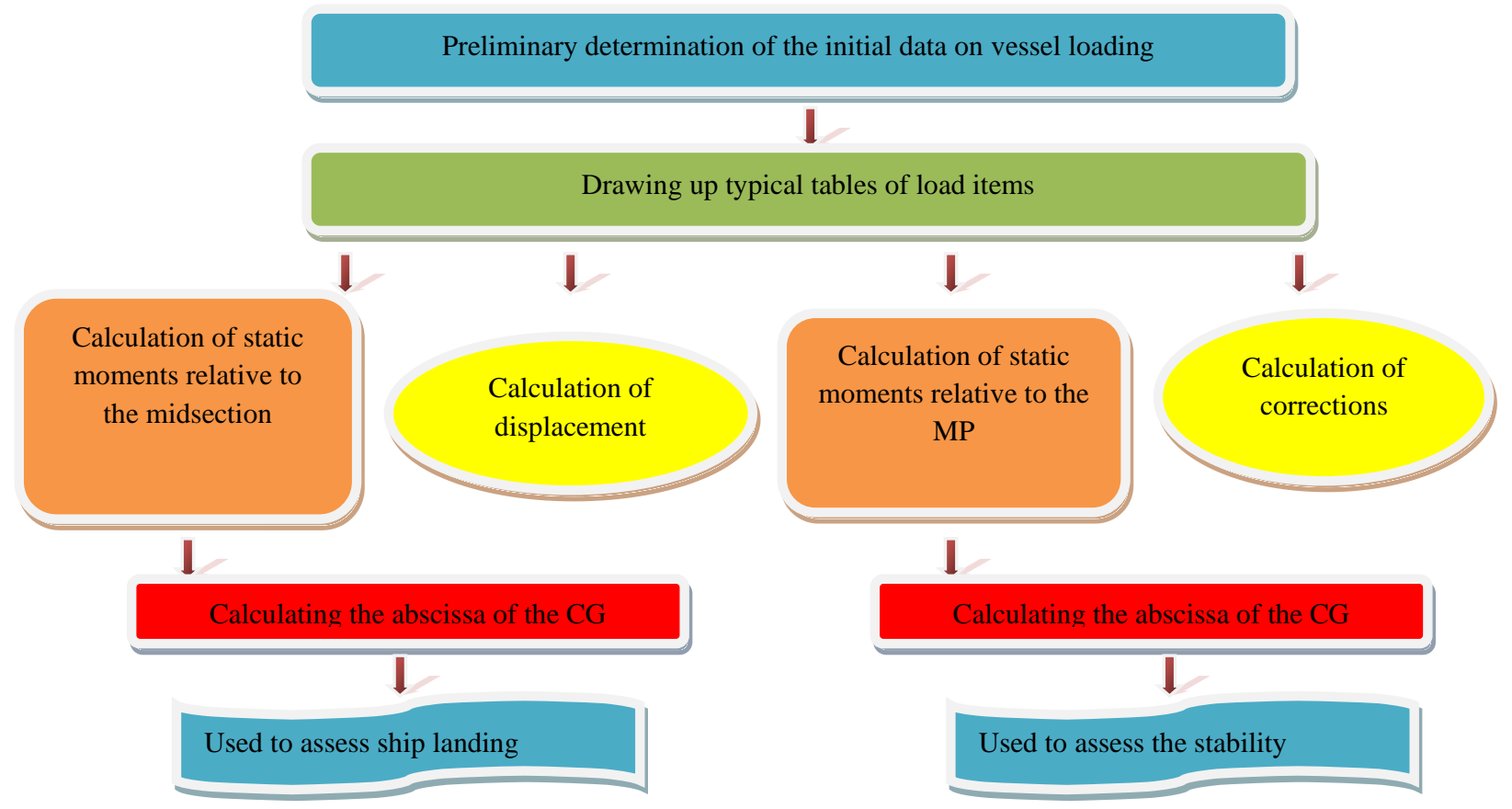

\section{REFERENCES}

1. Nguyen V. H. Method for determining the mass and coordinates of the center of gravity of a light ship with a wooden hull / Viet Hoan Nguyen. // Young scientist. - 2010. - No. 1. P. 94-100.

2. Sokolenko V. I. The navigation device for converting the coordinates of the satellite antenna of the vessel to the center of gravity / V. I. Sokolenko, S. E. Maltsev, A. S. Maltsev. // Materials of the scientific and technical conference "Transport technologies (sea and river fleets): infrastructure, navigation, transportation, automation". - 2018. - No. 28. - P. 210-221.

3. Tovstokoryi O.N Experimental determination of position of pivot point by assistance of Doppler lag / O.N Tovstokoryi, S.E Maltsev. // Materials of the scientific and technical conference "Transport technologies (sea and river fleets): infrastructure, navigation, transportation, automation". - 2016. No. 26. - P. 183-190.

4. Baigunusov V.B To navigators on the buoyancy and stability of the vessel / V.B Baigunusov. - Petropavlovsk-Kamchatski:
KamchatGTU, 2001. - 86 p.

5. Filimonov V.N Influence of a free surface on stability [Electronic resource] / V.N Filimonov // Baltic Lloyd. - 2020. Mode of access to the resource: https://baltlloyd.ru/morskaja-praktika/ostojchivost/vlijanie-svobodnojpoverhnosti-na-ostojchivost.

6. Bessonov M. Static moment of the cross-sectional area [Electronic resource] / Mikhail Bessonov // dx / dy. - 2015. - Mode of access to the resource: http://dx-dy.ru/.

7. Static moment of the section [Electronic resource] // Theory and problem solving. - 2009. - Mode of access to the resource: https://isopromat.ru/sopromat/teoria/staticheskijmoment-ploshadi.

8. Sizov V. G. The theory of the ship / V. G. Sizov. - Odessa: FENZHS, 2003. - $284 \mathrm{p}$.

9. Chapter II-2-Design - Fire protection, detection and extinguishing of fires - Rule 3 [Electronic resource] // SOLAS. 1974. - Mode of access to the resource: https://zakon.rada.gov.ua/laws/show/995_251. 